Draft VERsion MAY 4, 2007

Preprint typeset using $\mathrm{LATEX}_{\mathrm{E}}$ style emulateapj v. 10/09/06

\title{
PHOTODESORPTION OF CO ICE
}

\author{
Karin I. Öвerg ${ }^{1 *}$, Guido W. Fuchs ${ }^{1}$, Zainab Awad ${ }^{1}$, Helen J. Fraser ${ }^{2}$, Stephan Schlemmer $^{3}$, Ewine F. van \\ DishoECK $^{4}$ AND HAROLd LiNNARTZ ${ }^{1}$ \\ Draft version May 4, 2007
}

\begin{abstract}
At the high densities and low temperatures found in star forming regions, all molecules other than $\mathrm{H}_{2}$ should stick on dust grains on timescales shorter than the cloud lifetimes. Yet these clouds are detected in the millimeter lines of gaseous CO. At these temperatures, thermal desorption is negligible and hence a non-thermal desorption mechanism is necessary to maintain molecules in the gas phase. Here, the first laboratory study of the photodesorption of pure CO ice under ultra high vacuum is presented, which gives a desorption rate of $3 \times 10^{-3} \mathrm{CO}$ molecules per UV $(7-10.5 \mathrm{eV})$ photon at 15 $\mathrm{K}$. This rate is factors of $10^{2}-10^{5}$ larger than previously estimated and is comparable to estimates of other non-thermal desorption rates. The experiments constrains the mechanism to a single photon desorption process of ice surface molecules. The measured efficiency of this process shows that the role of CO photodesorption in preventing total removal of molecules in the gas has been underestimated.
\end{abstract}

Subject headings: Molecular data — Molecular processes — ISM: abundances — Physical Data and Processes: astrochemistry — ISM: molecules

\section{INTRODUCTION}

In the cold and dense interstellar regions in which stars are formed, $\mathrm{CO}$ and other molecules collide with and stick to cold (sub)micron-sized silicate particles, resulting in icy mantles (Léger et al. 1985; Boogert \& Ehrenfreund 2004). Chemical models of these regions show that all molecules except for $\mathrm{H}_{2}$ are removed from the gas phase within $\sim 10^{9} / n_{\mathrm{H}}$ years, where $n_{\mathrm{H}}$ is the total hydrogen number density (Willacy \& Millar 1998). For a typical density of $10^{4} \mathrm{~cm}^{3}$, this time scale is much shorter than the estimated age of such regions and hence molecules like $\mathrm{CO}$ should be completely frozen out in these clouds. Yet, these clouds are detected in the millimeter lines of gaseous CO (Bergin et al. 2001, 2002). Similarly cold CO gas has been detected in the midplanes of protoplanetary disks (Dartois et al. 2003). A recent study of several disks (Piétu et al. 2007) even finds that the bulk of the gaseous $\mathrm{CO}$ is at temperatures lower than $17 \mathrm{~K}$, below the condensation temperature onto grains. Thus some desorption mechanism is needed to keep part of the $\mathrm{CO}$ and other molecules in the gas phase. Clarifying this desorption mechanism is important in understanding the physical and chemical evolution of interstellar clouds. Because the sticking probability of even volatile species like $\mathrm{CO}$ has been shown to be unity (Bisschop et al. 2006), it is the desorption mechanism and rate that controls the allocation of molecules between gas and solid phase. This allocation of molecules affects the gas phase and surface reactions as well as the

\footnotetext{
* To whom correspondence should be addressed; E-mail: oberg@strw.leidenuniv.nl.

${ }^{1}$ Sackler Laboratory for Astrophysics, Leiden Observatory, University of Leiden, P.O. Box 9513, NL 2300 RA Leiden, The Netherlands.

2 Department of Physics, Scottish Universities Physics Alliance (SUPA), University of Strathclyde, John Anderson Building, 107 Rottenrow East, Glasgow G4 ONG, Scotland.

3 Physikalisches Institut, Universität zu Köln, Zülpicher Str. 77, 50937 Cologne, Germany

${ }^{4}$ Leiden Observatory, University of Leiden, P.O. Box 9513, NL 2300 RA Leiden, The Netherlands.
}

dust properties.

The case of $\mathrm{CO}$ is of particular importance, as it is the most common molecule after $\mathrm{H}_{2}$ and the prime tracer of molecular gas. It is also a key constituent in the formation of more complex and pre-biotic species (Tielens \& Charnlev 1997), and its partitioning between the grain and gas phase therefore has a large impact on the possible chemical pathways (van Dishoeck 2006). In dense clouds without embedded energy sources, the grain temperature is low enough, around $10 \mathrm{~K}$, that thermal desorption is negligible and hence desorption must occur through photon or cosmic ray induced processes. External UV photons from the interstellar radiation field can penetrate into the outer regions of dense clouds and cosmic rays are always present, even in the most shielded regions.

Photodesorption has been proposed as an important desorption pathway of ices in protoplanetary disks and other astrophysical regions with dense clumps of material and excess UV photons (Willacy \& Langer 2000; Dominik et al. 2005). The lack of experimentally determined photodesorption rates for most astrophysically relevant molecules and conditions has prevented progress in this area, however, and theoretical estimates range by orders of magnitude, with desorption rates from $10^{-5}$ to $10^{-8}$ CO molecules UV-photon ${ }^{-1}$ (Draine \& Salpeter 1979; Hartquist \& Williams 1990). Due to this low estimated rate, $\mathrm{CO}$ photodesorption has generally been regarded as an insignificant process in astrophysical environments.

As the present study shows, this assumption is not correct, and the actual desorption rate is at least two orders of magnitude larger than the previous high estimate. Here, we present the results of an experimental study under astrophysically relevant conditions of the photodesorption rate of $\mathrm{CO}$ ice and of the mechanism involved.

\section{EXPERIMENTS}


The experimental set-up has been described in detail elsewhere (Fuchs et al. 2006). In these experiments, thin ices of 2 to 350 monolayers (ML) are grown at $15 \mathrm{~K}$ on a gold substrate under ultra-high vacuum conditions $\left(P<10^{-10} \mathrm{mbar}\right)$. The ice films are subsequently irradiated at normal incidence with UV light from a broadband hydrogen microwave discharge lamp, which peaks around $125 \mathrm{~nm}$ and covers $120-170 \mathrm{~nm}(7-10.5 \mathrm{eV})$ (Muñoz Caro \& Schutte 2003). The lamp has a UV photon flux, measured with a NIST calibrated silicon diode, of $(6 \pm 2) \times 10^{13}$ photons $\mathrm{s}^{-1} \mathrm{~cm}^{-2}$ at the substrate surface in its standard setting. The emission resembles the spectral distribution of the UV interstellar radiation field that impinges externally on all clouds as well as that of the UV radiation produced locally inside clouds by the decay of electronic states of $\mathrm{H}_{2}$, excited by energetic electrons resulting from cosmic-ray induced ionization of hydrogen (Sternberg et al. 1987). The setup allows simultaneous detection of molecules in the gas phase by quadrupole mass spectrometry (QMS) and in the ice by reflection absorption infrared spectroscopy (RAIRS) using a Fourier transform infrared spectrometer.

Once an ice is grown, it remains stable until it is UV irradiated. The layer thickness of the ice is monitored by recording RAIR spectra (Fig. 1). The intensity of the CO RAIRS profile is linearly correlated with the layer thickness of the $\mathrm{CO}$ ice up to $\sim 20$ monolayers (ML). One monolayer is generally taken to consist of $\sim 10^{15}$ molecules $\mathrm{cm}^{-2}$ and the rate of the $\mathrm{CO}$ photodesorption is subsequently derived from the intensity loss in the RAIR spectra as function of time (Fig. 2). From this loss of ice molecules and the known photon flux hitting the surface it is possible to calculate the desorption rate as the number of molecules desorbed per incident photon. Re-condensation will play a negligible role given the small surface area of the sample and the resulting unterestimate of the actual photodesorption will be substantially lower than other sources of inaccuracy. Above 20 ML the photodesorption rate can no longer be calculated from the RAIRS profile as the integrated absorbance of the peak is no longer linearly dependent on the number of molecules. Instead a relative photodesorption rate can be determined by mass spectrometry of the desorbed gas phase molecules. This rate is converted to an absolute photodesorption rate by comparison with thin layer experiments where both QMS and RAIRS data are available. Simultaneous mass spectrometry of gas phase constituents shows that only $\mathrm{CO}$ molecules are desorbed. Furthermore the RAIRS results show that no other molecules are formed during the UV irradiation (i.e. less than $0.2 \%$ of the $\mathrm{CO}$ ice is converted to $\mathrm{CO}_{2}$ after 8 hours of irradiation of $8 \mathrm{ML} \mathrm{CO}$ ice). This result is of importance as in traditional vacuum experiments with substantially thicker and less pure ices, reaction products have been identified upon UV photoprocessing (Loeffler et al. 2005) and this may affect the photodesorption efficiency.

In these experiments the thickness of the ice, which is needed to determine the desorption rate, was calculated from the observed difference in desorption from multilayer coverages (constant rate) and monolayer coverages (decreasing rate). From the RAIR spectra at this turning point, the integrated absorbance of $1 \mathrm{ML}$ is estimated to within $20 \%$. The original thickness of the ice can then be calculated from the integrated absorbance of the RAIRS feature before onset of desorption. This technique is based on the assumption that the ice is quite flat, which is confirmed by the results of the experiments.

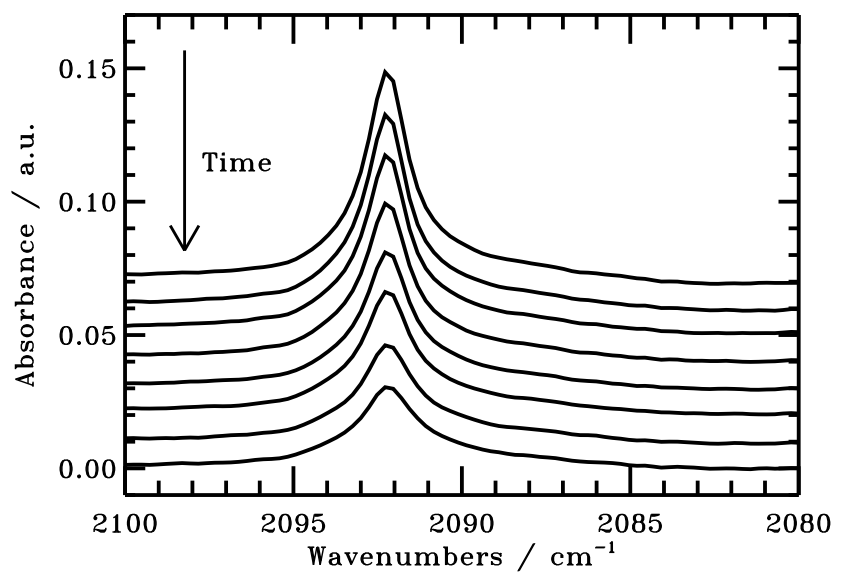

FIG. 1.- RAIR spectra of the $\mathrm{C}^{18} \mathrm{O} \nu=1-0$ vibrational band at $2040 \mathrm{~cm}^{-1}\left(2140 \mathrm{~cm}^{-1}\right.$ for normal CO) acquired before irradiation of a $8 \mathrm{ML}$ of $\mathrm{C}^{18} \mathrm{O}$ ice and then after every hour of irradiation during 8 hours. The drop in integrated absorbance of the $\mathrm{C}^{18} \mathrm{O}$ ice band is linear with UV irradiation time. In most of our experiments we used the $\mathrm{C}^{18} \mathrm{O}$ isotopologue instead of $\mathrm{C}^{16} \mathrm{O}$ to rule out any unwanted contributions from outgassing of the vacuum chamber. Control experiments with $\mathrm{C}^{16} \mathrm{O}$ resulted in the same photodesorption rate within our experimental uncertainty.

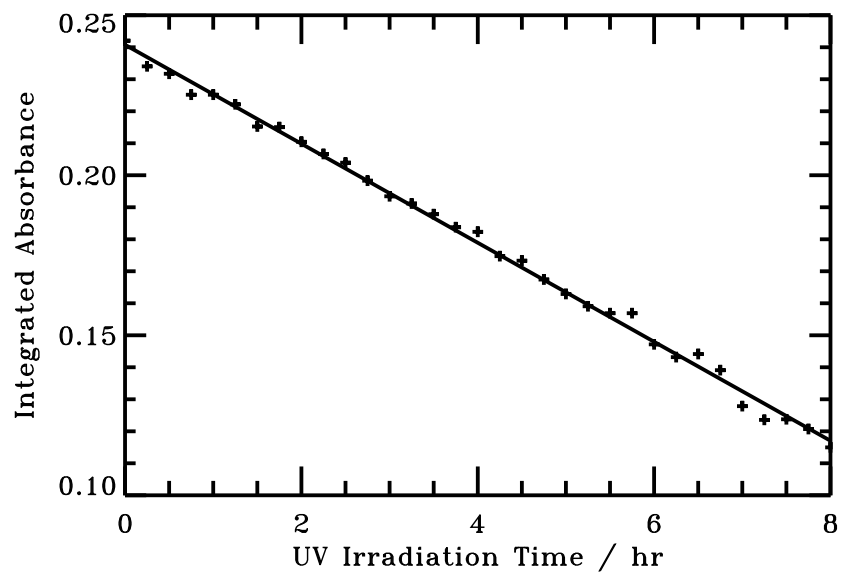

FIG. 2.- The integrated absorbance of the CO RAIRS band acquired before irradiation of a $8 \mathrm{ML} \mathrm{C}{ }^{18} \mathrm{O}$ ice and then after every 15 minutes of UV irradiation during 8 hours. The photodesorption rate is calculated from the slope of the fitted line. The fitted line gives a photodesorption rate in loss of integrated absorbance in integrated absorbance units (I.A.U.) per hour (here 0.015 I.A.U. $\mathrm{hr}^{-1}$ ). The amount of integrated absorbance per monolayer can be derived from the integrated absorbance of 0.24 I.A.U. of $8 \mathrm{ML}$ at time 0 . Using the known $\mathrm{UV}$ flux and $\mathrm{CO}$ coverage, the loss of integrated absorbance per hour is converted to a photodesorption rate in $\mathrm{CO}$ molecules per UV (7-10.5 eV) photon: $\quad R_{\mathrm{pd}}=(0.015$ I.A.U. $/ \mathrm{hr}) \times(\mathrm{hr} / 3600 \mathrm{~s}) \times(8 \mathrm{ML} / 0.24$ I.A.U. $)$

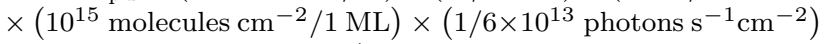
$=0.003$ molecules photon $^{-1}$

\section{RESULTS}

The evaluation of the experiments results in a constant rate of $(3 \pm 1) \times 10^{-3} \mathrm{CO}$ molecules photon ${ }^{-1}$, averaged 
over the wavelength range of the lamp. This rate is fully reproducible from repeated experiments of $8 \mathrm{ML}$ coverage and has a standard deviation of $\sim 15 \%$ (Fig. 3). The uncertainty in the absolute value is somewhat larger, up to $30 \%$, dominated by the uncertainty in the UV photon flux and coverage.

The thickness of the $\mathrm{CO}$ ice has been varied between 2 and $350 \mathrm{ML}$. We find that the photodesorption rate of $\mathrm{CO}$ is independent of the ice thickness (Fig. 3). This suggests that only the upper layers are involved in the photodesorption event. It is also consistent with a surface that is quite smooth, since at 2 ML the entire surface must still be covered to achieve the same photodesorption rate as for $350 \mathrm{ML}$. To confirm that the desorbed molecules only originate from the top layers we performed experiments with two layers of ices comprising different $\mathrm{CO}$ isotopologues. When $2 \mathrm{ML}$ of $\mathrm{C}^{18} \mathrm{O}$ is deposited on top of $8 \mathrm{ML}$ of $\mathrm{C}^{16} \mathrm{O}$ the desorption rate from the bottom layer drops with less than $20 \%$. In contrast depositing $4 \mathrm{ML}$ of $\mathrm{C}^{18} \mathrm{O}$ on top of $8 \mathrm{ML}$ of $\mathrm{C}^{16} \mathrm{O}$ ice reduces the $\mathrm{C}^{16} \mathrm{O}$ desorption rate with more than $80 \%$. This confirms that mainly the top few layers of the CO ice are directly involved. Furthermore, the CO photodesorption rate is directly proportional to the photon flux within the flux range covered here $\left((4-8) \times 10^{13}\right.$ photons $\left.\mathrm{s}^{-1} \mathrm{~cm}^{-2}\right)$.

In contrast to previous findings on $\mathrm{H}_{2} \mathrm{O}$ photodesorption (Westley et al. 1995), we find that the CO photodesorption rate is independent of the total photon dose as well as the irradiation time, as long as 1 ML is left on the surface. The mass signals show an onset of the photodesorption within the time constant of our QMS system (a few seconds) when the UV source is turned on.

In addition to the experiments on $\mathrm{CO}$ ices, a thin layer of $\mathrm{N}_{2}$ ice $(8 \mathrm{ML})$ was irradiated under the same conditions as the $\mathrm{CO}$ ices with the aim to compare the two photodesorption rates. It is found that $\mathrm{N}_{2}$ has no detectable photodesorption in the present set-up, which puts an upper bound to the photodesorption rate of pure $\mathrm{N}_{2}$ ice of $2 \times 10^{-4}$ molecules UV-photon ${ }^{-1}$.

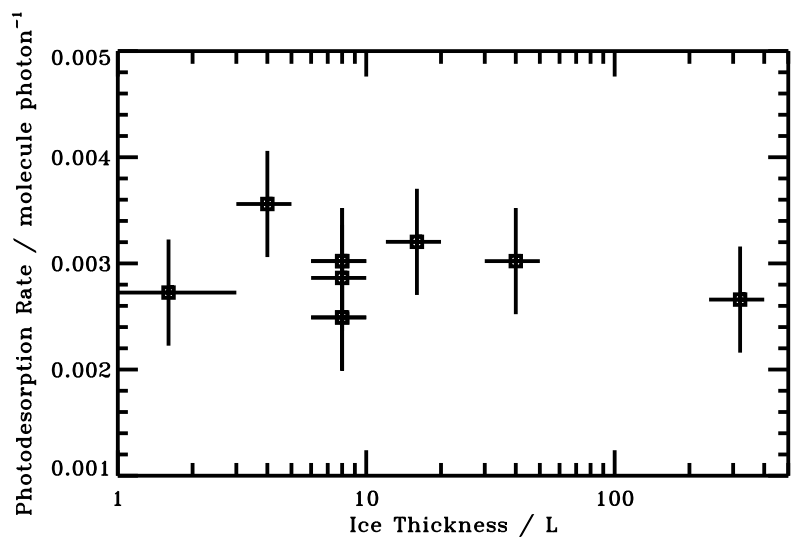

FIG. 3.- The desorption rate of $\mathrm{CO}$ at different layer thicknesses. From repeated experiments around $8 \mathrm{ML}$ the standard deviation in the photodesorption rate was determined, indicated by the size of the error bars. Within the experimental uncertainty derived from this spread, we conclude that the $\mathrm{CO}$ photodesorption rate is independent of the thickness of the CO ice.

\section{DISCUSSION}

\subsection{Photodesorption Mechanism}

The above experiments can be used to constrain the $\mathrm{CO}$ photodesorption mechanism. Its insensitivity to layer thickness (demonstrated in Fig. 3) indicates that only molecules from the top layers of the ice contribute to the photodesorption flux. In addition, it suggests that the CO photodesorption mechanism at these UV wavelengths does not involve the substrate. The linear dependence of the photodesorption rate on the UV intensity is not consistent with that the desorption is due to sublimation caused by heating of the ice as a whole. Together these results suggest that CO photodesorption occurs through a single photon-process, which is further supported by the immediate on-set of the desorption once the UV lamp is turned on. The opposite conclusion has previously been drawn for $\mathrm{H}_{2} \mathrm{O}$ (Westley et al. 1995).

The final support for a single-photon process is given by the different desorption rates for $\mathrm{CO}$ and $\mathrm{N}_{2}$ ice. The two molecules have similar inter-molecular binding energies and ice structures (Fuchs et al. 2006), which suggests that any difference in photodesorption rate must involve the internal structure of the molecules. A relevant difference between the two species is that CO has an electric dipole allowed transition in the vacuum ultraviolet $(7-10 \mathrm{eV}$ ) (Mason et al. 2006), exactly where the hydrogen lamp simulates the interstellar radiation field, while $\mathrm{N}_{2}$ does not. This transition corresponds to the solid state equivalent of the $\mathrm{A}^{1} \Pi-X^{1} \Sigma^{+}$electronic excitation of gaseous $\mathrm{CO}$ and the most plausible photodesorption mechanism hence involves this transition. After UV absorption, the excited molecule relaxes via a radiationless transition into vibrationally excited states of the electronic ground state which subsequently transfer part of this intramolecular energy to the weak intermolecular bonds with neighboring $\mathrm{CO}$ molecules, resulting in a desorption event. This desorption event may consist of more than the originally excited molecule desorbing, but experiments with mixed $\mathrm{CO} / \mathrm{N}_{2}$ ices are necessary to constrain this part of the mechanism in more detail.

\subsection{Astrophysical Implications}

The single photon mechanism of CO photodesorption means that the rate derived from these experiments can be easily applied to astrophysical environments without concerns about ice thicknesses and irradiation field strengths. It is illustrative to compare the photodesorption rate with other possible desorption routes in dark clouds. Since thermal desorption is negligible, species can only be kept in the gas phase through ice desorption induced by UV photons and cosmic rays. While cosmic rays have been proposed to directly desorb ices (Léger et al. 1985), the efficiency of heating an entire grain to the required desorption temperature rapidly drops with grain size so that usually only spot heating at an estimated rate of 70 molecules $\mathrm{cm}^{-2} \mathrm{~s}^{-1}$ is considered as a viable mechanism. The cosmic rays also produce UV photons so that the total photodesorption rate depends on both the external interstellar radiation field and the UV field produced inside the cloud by the cosmic rays. For a typical galactic cosmic ray flux, the resulting UV photon flux is of the order of $10^{4}$ photons $\mathrm{cm}^{-2} \mathrm{~s}^{-1}$ with a factor of 3 uncertainty (Shen et al. 2004). 
We calculated the desorption rate due to photodesorption in a dark cloud and compared this with the desorption due to spot-heating from Shen et al. (2004). Equations 1 and 2 describe the photodesorption rates of $\mathrm{CO}$ molecules from grain surfaces in molecules $\mathrm{cm}^{-2} \mathrm{~s}^{-1}$ due to external and cosmic ray induced UV photons, respectively, where $I_{\text {ISRF-FUV }}=1 \times 10^{8}$ photons $\mathrm{cm}^{-2} \mathrm{~s}^{-1}$ is the strength of the external irradiation field with energies 6-13.6 eV, $I_{\mathrm{CR}-\mathrm{FUV}}$ the strength of the UV field due to cosmic rays, $\gamma$ is a measure of $\mathrm{UV}$ extinction relative to visual extinction, which is $\sim 2$ for small interstellar grains (Roberge et al. 1991), and $Y_{\mathrm{pd}}$ is the experimentally determined photodesorption rate.

$$
\begin{gathered}
R_{\mathrm{UV}-\mathrm{PD}}=I_{\mathrm{ISRF}-\mathrm{FUV}} e^{-\gamma A_{\mathrm{V}}} Y_{\mathrm{pd}} \\
R_{\mathrm{CR}-\mathrm{PD}}=I_{\mathrm{CR}-\mathrm{FUV}} Y_{\mathrm{pd}}
\end{gathered}
$$

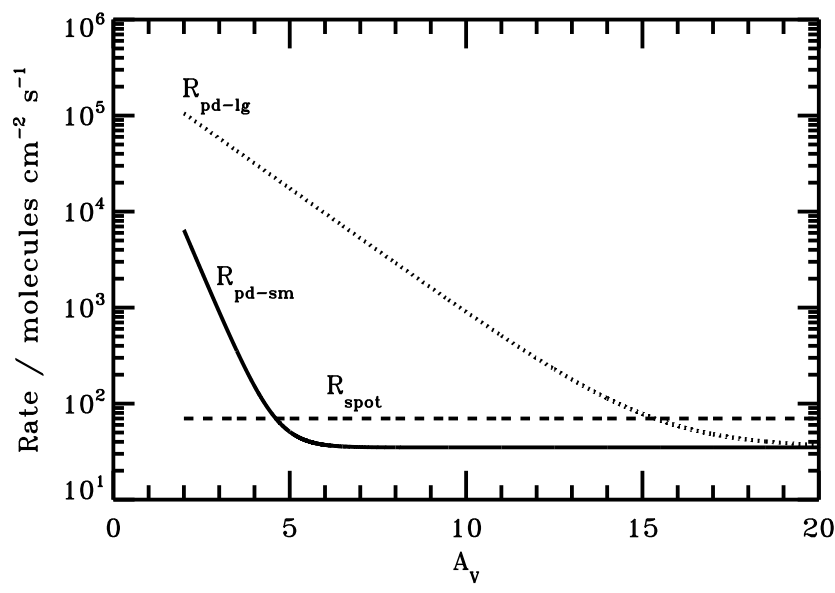

FIG. 4. - The photodesorption rate of CO for small $(0.1 \mu \mathrm{m}$, $\mathrm{R}_{\mathrm{pd}-\mathrm{sm}}$, full line) and large (a few $\mu \mathrm{m}, \mathrm{R}_{\mathrm{pd}-\mathrm{lg}}$, dotted line) grains compared to desorption due to spot heating by cosmic rays $\left(R_{\text {spot }}\right.$, dashed line). For small grains, applicable to dark clouds, the spot heating and photodesorption rates are comparable. When grains have grown to a few $\mu \mathrm{m}$ size the photodesorption dominates up to $A_{V}=15$.

Applying this model shows that photodesorption dominates at the edge of the cloud and becomes comparable (within the uncertainties of a few) to spot heating in the interior of a cloud, i.e. beyond a depth corresponding to an extinction of 3-4 $\mathrm{A}_{V}$ (Fig. 4). In the interior of the cloud the photodesorption rate due to cosmic rays is $\sim 30$ molecules $\mathrm{cm}^{-2} \mathrm{~s}^{-1}$, which is equivalent to $\sim 10^{-8}$ molecules grain ${ }^{-1} \mathrm{~s}^{-1}$ for grains with a $0.1 \mu \mathrm{m}$ radius. A rate of this magnitude may on its own explain the gas phase CO seen in dark clouds (Bergin et al. 2006). In comparison with the other plausible non-thermal desorption mechanisms, photodesorption has the advantage that the rate can now be determined experimentally and unambiguously included in astrophysical models.

One particularly interesting application of our derived photodesorption rate is to the case of $\mathrm{CO}$ in protoplanetary disks, where large abundances of cold CO-gas is observed. Aikawa \& Nomura (2006) argue that the cold CO-gas can be explained by vertical mixing and Semenov et al. (2006) by a combination of radial and vertical mixing of disk material. With the high photodesorption rate of $\mathrm{CO}$ derived here, non-thermal desorption of CO may suffice to explain these observations. An important characteristic of disks compared to dense clouds is dust coagulation, which reduces the absorption of the external UV field since larger grains absorb less efficiently at short wavelengths. In Eq. 1 this corresponds to $\gamma \leq 0.6$ (van Dishoeck et al. 2006), assuming the grains have grown to at least $\mu \mathrm{m}$ size as indicated by infrared observations of the silicate feature from the surfaces layers of the inner disk (e.g. Bouwman et al. 2001) and by millimeter observations of the outer disk showing growth up to $\mathrm{mm}$ size (e.g. Rodmann et al. 2006). The photodesorption rate due to external photons then dominates over other non-thermal desorption rates up to $\mathrm{A}_{V}=15$, but detailed modeling is necessary to determine whether this rate is high enough to offer an alternative to the turbulence theory in explaining the observed $\mathrm{CO}$ gas. It is clear, however, that photodesorption can no longer be ignored in astrophysical models and may explain a large part of the gas observed in cold and dense regions.

We thank F.A. van Broekhuizen and W.A. Schutte for initial work on our photodesorption instrument. Funding was provided by NOVA, the Netherlands Research School for Astronomy, a grant from the European Early Stage Training Network ('EARA' MEST-CT-2004-504604) and a NWO Spinoza grant.

\section{REFERENCES}

Aikawa, Y. \& Nomura, H. 2006, ApJ, 642, 1152

Bergin, E. A., Alves, J., Huard, T., \& Lada, C. J. 2002, ApJL, 570, L101

Bergin, E. A., Ciardi, D. R., Lada, C. J., Alves, J., \& Lada, E. A. 2001, ApJ, 557, 209

Bergin, E. A., Maret, S., van der Tak, F. F. S., Alves, J., Carmody, S. M., \& Lada, C. J. 2006, ApJ, 645, 369

Bisschop, S. E., Fraser, H. J., Öberg, K. I., van Dishoeck, E. F., \& Schlemmer, S. 2006, A\&A, 449, 1297

Boogert, A. C. A. \& Ehrenfreund, P. 2004, in ASP Conf. Ser. 309: Astrophysics of Dust, ed. A. N. Witt, G. C. Clayton, \& B. T. Draine, 547-572

Bouwman, J., Meeus, G., de Koter, A., Hony, S., Dominik, C., \& Waters, L. B. F. M. 2001, A\&A, 375, 950

Dartois, E., Dutrey, A., \& Guilloteau, S. 2003, A\&A, 399, 773

Dominik, C., Ceccarelli, C., Hollenbach, D., \& Kaufman, M. 2005, ApJL, 635, L85

Draine, B. T. \& Salpeter, E. E. 1979, ApJ, 231, 438
Fuchs, G. W., Acharyya, K., Bisschop, S. E., Öberg, K. . I., van Broekhuizen, F. A., Fraser, H. J., Schlemmer, S., van Dishoeck, W. F., \& Linnartz, H. 2006, Faraday Discussions, 133, 331

Hartquist, T. W. \& Williams, D. A. 1990, Mon. Not. R. astr. Soc., 247,343

Léger, A., Jura, M., \& Omont, A. 1985, A\&A, 144, 147

Loeffler, M. J., Baratta, G. A., Palumbo, M. E., Strazzulla, G., \& Baragiola, R. A. 2005, A\&A, 435, 587

Mason, N. J., Dawes, A., Holtom, P. D., Mukerji, R. J., Davis, M. P., Sivaraman, B., Kaiser, R. I., Hoffmann, S. V., \& Shaw, D. A. 2006, Faraday Discussions, 133, 1

Muñoz Caro, G. M. \& Schutte, W. A. 2003, A\&A, 412, 121

Piétu, V., Dutrey, A., \& Guilloteau, S. 2007, ArXiv Astrophysics e-prints

Roberge, W. G., Jones, D., Lepp, S., \& Dalgarno, A. 1991, ApJS, 77,287

Rodmann, J., Henning, T., Chandler, C. J., Mundy, L. G., \& Wilner, D. J. 2006, A\&A, 446, 211 
Semenov, D., Wiebe, D., \& Henning, T. 2006, ApJ, 647, L57

Shen, C. J., Greenberg, J. M., Schutte, W. A., \& van Dishoeck, E. F. 2004, A\&A, 415, 203

Sternberg, A., Dalgarno, A., \& Lepp, S. 1987, ApJ, 320, 676

Tielens, A. G. G. M. \& Charnley, S. B. 1997, Origins of Life and Evolution of the Biosphere, 27, 23

van Dishoeck, E. F. 2006, Proceedings of the National Academy of Science, 103, 12249 van Dishoeck, E. F., Jonkheid, B., \& van Hemert, M. C. 2006, Faraday Discussions, 133, 231

Westley, M. S., Baragiola, R. A., Johnson, R. E., \& Baratta, G. A. 1995, Nature, 373, 405

Willacy, K. \& Langer, W. D. 2000, ApJ, 544, 903

Willacy, K. \& Millar, T. J. 1998, MNRAS, 298, 562 